\title{
Ergonomie am Behandlungsplatz Warum sich intuitive Arbeitswege lohnen und Rückenschmerzen nicht sein müssen*
}

Körperliche Beschwerden nach einem langen Behandlungstag gehören wahrlich nicht zu den liebsten Begleiterscheinungen eines Berufs - und doch treten sie häufiger auf als geahnt. Kaum eine Berufsgruppe übt ihre Tätigkeit in einer derart einseitigen und unnatürlichen Haltung aus wie die Zahnärzte. Die Folgen: Rund 64\% klagen über Rückenschmerzen und über $42 \%$ über haltungsbedingte Kopfschmerzen. * *

Dass diese Beschwerden vermeidbar sind, stellte der amerikanische Zahnarzt Dr. Daryl Beach bereits Anfang der 50er Jahre fest. Er entwickelte ein Behandlungskonzept, auch „12-Uhr-Behandlung“ genannt, das in erster Linie auf einer natürlichen und stabilen Körperhaltung basiert, bei der lediglich die Unterarme aktiv sind. Beobachtet hatte Dr. Beach diese Haltung bei Versuchspersonen, die „blind“ eine kontrollierte sensomotorische Präzisionsarbeit (z.B. Schälen eines Apfels) ausführen sollten und alle instinktiv diese natürliche und stabile Haltung einnahmen.

Übertragen auf den zahnärztlichen Alltag bedeutet dies: Nicht etwa der Zahnarzt beugt sich - mit verdrehtem Rücken - über den Patienten. Vielmehr wird der Kopf des Patienten, der entspannt in einer speziell geformten Behandlungsein-
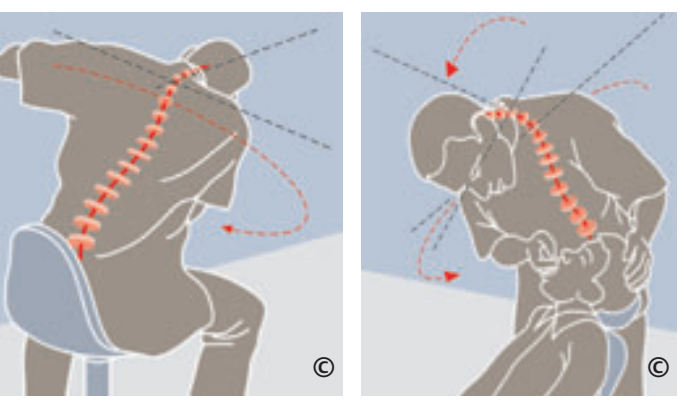

Rückenprobleme sind bei längerer Beibehaltung dieser unnatürlichen Haltung (links) vorprogrammiert. Auch diese Beugehaltung (rechts) belastet den Rücken übermäßig und sorgt für frühzeitigen Verschleiß.

* Nach dem ergonomischen Konzept von Dr. Daryl Beach

** It. einer Untersuchung des Freien Verbandes Deutscher Zahnärzte heit liegt, in die jeweils gewünschte Stellung gedreht. Der Zahnarzt kann dadurch in seiner natürlichen Haltung bleiben.

- Die Belastungen des Zahnarztes durch unnatürliche Fehlhaltungen und damit einhergehender körperlicher Verschleiß werden auf ein Minimum reduziert. normalerweise nicht mehr auf.

- Die natürlichen und effizienten Behandlungsbewegungen erlauben ein präziseres und sichereres Arbeiten auch in schwer zugänglichen Bereichen.

- Die ausgewogene natürliche Haltung sorgt für maximale Konzentration und Präzision.

- Die ruhigen Bewegungen des Zahnarztes führen zu einer entspannteren und stressfreien Atmosphäre.

\section{Die optimale Arbeitshaltung ist zugleich auch intuitiv}

Voraussetzung für ein körperschonendes Arbeiten ist die optimale Haltung des gesamten Körpers. Dr. Beach spricht in dem Zusammenhang von einer instinktiven, natürlichen und ausgewogenen Arbeitshaltung oder auch Referenzhaltung.

Er nennt sie instinktiv, da alle Menschen bei Präzisionsarbeiten automatisch und unbewusst diese Position einnehmen.

Die Natürlichkeit dieser Haltung legen bereits die Skelettstruktur und der Muskeltonus physiologisch und anatomisch fest. Und da die Kräfte der Schwerkraft hier in einem stabilen Gleichgewicht sind, ist die Haltung zudem ausgewogen. In der praktischen Umsetzung sieht das wie folgt aus: Während der Behandlung
- Rücken- und Kopfschmerzen treten nimmt man eine sitzende Körperhaltung ein, bei der sich die Sitzhöhe auf Kniehöhe befindet. Die Füße stehen flach auf dem Boden. Während die Unterschenkel in einer vertikalen Position sind, neigt man die Oberschenkel leicht und bildet damit einen Winkel von ca. $30^{\circ}$. Die Längsachse des Torsos ist aufrecht. Oberarme und Ellbogen folgen der Schwerkraft und hängen locker herab, die Ellbogen stehen nicht ab und der Unterarm ist vor dem Körper. Die Fingerspitzen befinden sich am Behandlungspunkt in der mittleren Sagittalebene, in der Höhe des Herzens. Den Kopf neigt man leicht nach vorne, sodass die Interpupillar-Linie horizontal ausgerichtet ist. Diese Referenzhaltung lässt sich mit dem Behandlungskonzept von J. Morita Europe stabil und bequem einnehmen. Die Behandlungsserie Spaceline EMCIA wurde auf Basis des Dr.-Beach-Konzeptes entwickelt und

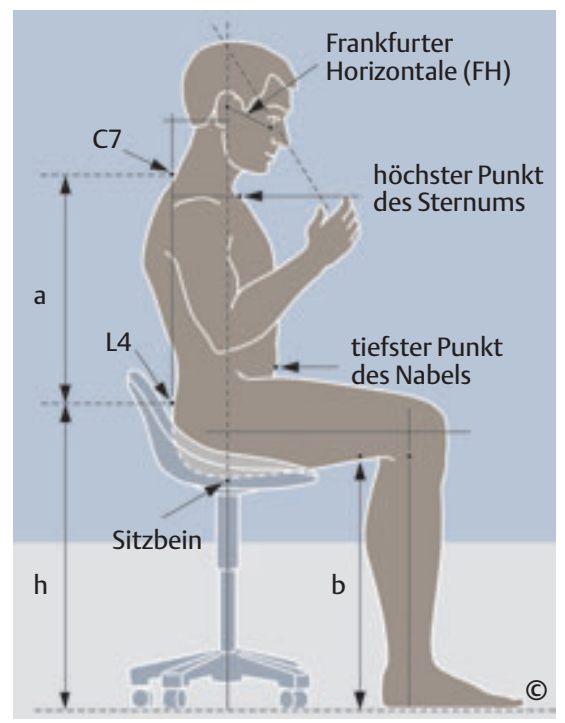

Der Rumpf ist in natürlicher Haltung aufrecht ohne Rotation oder Beugung. Die Linie zwischen C7 und L4 - siehe Linie a in der Abbildung - ist vertikal. Eine Linie durch den Nabel, die im rechten Winkel zur Linie a steht, verläuft parallel mit der Längsachse des Oberschenkels. Dies ist einer der Prüfpunkte für die „Referenzhaltung“. 
